[Article]

\title{
氮掺杂石墨烯的制备及其对氧还原反应的电催化性能
}

\author{
彭 三 郭慧林* 六晓峰 \\ (西北大学化学与材料科学学院, 合成与天然功能分子化学教育部重点实验室, 西安 710069)
}

\begin{abstract}
摘要: 以氧化石墨烯 (GO)为原料、丙酮肜(DMKO)为还原剂和氮掺杂剂, 采用化学还原法制备了不同氮掺杂含 量的石墨烯(NG). 利用场发射透射电子显微镜(FETEM)、紫外-可见(UV-Vis)光谱、傅里叶变换红外(FTIR)光谱、 $X$ 射线光电子能谱 (XPS)、zeta 电位和纳米粒度分析、循环伏安 (CV)和旋转圆盘电极 (RDE)等手段对材料的形 貌、结构、成分和电化学性质进行表征. 结果显示: DMKO 能有效地还原 GO, 且通过调节 GO 与 DMKO 的质量 比, 可以得到不同还原效果的 NG, 其氮含量范围为 $4.40 \%-5.89 \%$ (原子分数); GO 与 DMKO 的质量比为 $1: 0.7$ 时制备的氮掺杂石墨烯 $(N G-1)$ 在 $\mathrm{O}_{2}$ 饱和 $0.1 \mathrm{~mol} \cdot \mathrm{L}^{-1} \mathrm{KOH}$ 溶液中对氧还原反应(ORR)的电催化性能最佳, 其 ORR 峰电流为 $0.93 \mathrm{~mA} \cdot \mathrm{cm}^{-2}$, 电子转移数为 3.6 , 这归因于其较高含量的吡啶 $-\mathrm{N}$ 增加了材料的 ORR 活性位 点. 此外, 石墨化- $\mathrm{N}$ 由于其较高的电子导电性倾向于产生较高的氧还原峰电流, 而吡啶- $\mathrm{N}$ 较低的超电势倾向于 产生较正的氧还原峰电位. 与商用 $\mathrm{Pt} / \mathrm{C}$ 相比, 该材料展现出了优异的抗 $\mathrm{CH}_{3} \mathrm{OH}$ “跨界效应”的特性.
\end{abstract}

关键词: 石墨烯; 氮掺杂; 氧还原反应; 电催化

中图分类号: 0646

\section{Preparation of Nitrogen-Doped Graphene and Its Electrocatalytic Activity for Oxygen Reduction Reaction}

\author{
PENG San GUO Hui-Lin* KANG Xiao-Feng \\ (Key Laboratory of Synthetic and Natural Functional Molecule Chemistry (Ministry of Education), College of Chemistry \\ and Materials Science, Northwest University, Xi'an 710069, P. R. China)
}

\begin{abstract}
Nitrogen-doped graphene (NG) was prepared by chemical reduction of graphene oxide (GO) using dimethyl ketoxime (DMKO) as reducing and doping agents. The morphologies, structures, compositions, and electrocatalytic activities of the as-prepared materials were investigated using field-emission transmission electron microscopy (FETEM), ultraviolet-visible (UV-Vis) spectroscopy, Fourier-transform infrared (FTIR) spectroscopy, X-ray photoelectron spectroscopy (XPS), zeta potential and nanoparticle analyses, cyclic voltammetry (CV), and the rotating disk electrode (RDE) method. The results showed that GO sheets were effectively reduced by DMKO. NG samples with different nitrogen contents were obtained by adjusting the mass ratio of GO to DMKO; the nitrogen contents were in the range $4.40 \%-5.89 \%$ (atomic fraction). NG-1, obtained using a GO/DMKO mass ratio of 1:0.7, showed excellent electrocatalytic activity in the oxygen reduction reaction (ORR) in an $\mathrm{O}_{2}$-saturated $0.1 \mathrm{~mol} \cdot \mathrm{L}^{-1} \mathrm{KOH}$ solution. The peak current was $0.93 \mathrm{~mA} \cdot \mathrm{cm}^{-2}$, and the number of electrons transferred per $\mathrm{O}_{2}$ was 3.6; this was attributed to the increase in the number of ORR active sites in the presence of pyridinic-N. In addition, the electrocatalytic activity of NG was found to be dependent on the graphitic-N content, which determined the limiting current density, because of its higher electronic conductivity.
\end{abstract}

Received: May 15, 2014; Revised: July 10, 2014; Published on Web: July 11, 2014.

"Corresponding author. Email: hlguo@nwu.edu.cn; Tel/Fax: +86-29-88302604.

The project was supported by the National Natural Science Foundation of China (21175105, 21375104), Specialized Research Fund for the Doctoral Program of Higher Education of China (20126101110015), Natural Science Foundation of Shaanxi Province, China (2014JM2042), and State Key Laboratory of Analytical Chemistry for Life Science, China (SKLACLS1210).

国家自然科学基金(21175105, 21375104), 教育部高等学校博士学科点专项科研基金(20126101110015), 陕西省自然科学基金(2014JM2042)和 生命分析化学国家重点实验室开放基金(SKLACLS1210)资助项目

(C) Editorial office of Acta Physico-Chimica Sinica 
The pyridinic-N content improved the onset potential, because of its lower overpotential for the ORR. NG therefore exhibited a high selectivity in the ORR, with good tolerance of methanol cross-over effects. It is therefore superior to commercial Pt/C catalysts.

Key Words: Graphene; Nitrogen doping; Oxygen reduction reaction; Electrocatalysis

\section{1 引言}

随着全球能源危机的不断加剧, 探索并开发出 一种高效的新能源, 是人类走出能源困境, 走向可 持续发展道路的必经之路. 燃料电池, 作为一种直 接将物质的化学能转化为电能的电化学发电装置, 其在工作过程中不受卡诺循环的限制, 能量转化率 高达 40\%-60\%, 被认为是 21 世纪新型洁净、高效的 发电技术之一. 在燃料电池中, 阴极氧还原反应 (ORR) 对于能量转换扮演着至关重要的角色, 而 ORR 催化剂的活性和稳定性是衡量燃料电池性能 的重要指标. 目前, $\mathrm{Pt}$ 及其合金被认为是商用燃料 电池中催化效果最好的 ORR 电催化剂, ${ }^{1-3}$ 但 $\mathrm{Pt}$ 作为 一种贵金属, 根据美国能源部提出的 $5 \mathrm{~kW} \cdot \mathrm{g}^{-1}$ 金属 $\mathrm{Pt}$ 的负载量计算, $\mathrm{Pt}$ 催化剂的费用仍然占据整个燃 料电池系统全部费用的 $50 \%$ 左右. ${ }^{4}$ 且 Pt 在催化过程 中易受到 $\mathrm{CH}_{3} \mathrm{OH}$ “跨界效应”和 $\mathrm{CO}$ 中毒等因素的影 响而导致其催化效率下降. ${ }^{5}$ 因此, 发展一种同时具 有良好催化活性、稳定性和成本低廉的非 Pt 基催化 剂具有重要现实意义. 目前, 人们已经研究了碳纳 米材料、 ${ }^{6}$ 过渡金属 $(\mathrm{Fe} 、 \mathrm{Co} 、 \mathrm{Ru})$ 及其大环配合物材 料 4,7 作为 ORR 催化剂的性能.

石墨烯作为一种独特的 $s p^{2}$ 杂化的二维单层石 墨, ${ }^{8}$ 其稳定的六边形晶格结构赋予石墨烯许多优异 的物理和化学性质. ${ }^{9-14}$ 近年来, 人们发现将氮元素 引入碳材料可以提高催化剂的耐久性和稳定性, 并 且氮元素的掺杂可以改变碳材料的电负性, 使得氮 原子周围的碳原子带有更多的正电荷, 有利于氧气 的吸附活化, 进而促进氧气的还原. ${ }^{15}$ 与商用的铂炭 催化剂 $(\mathrm{Pt} / \mathrm{C})$ 相比, 氮掺杂石墨烯 $(\mathrm{NG})$ 不仅具有良 好的 ORR 活性和稳定性, ${ }^{16}$ 而且还具有优异的抗 $\mathrm{CH}_{3} \mathrm{OH}$ “跨界效应”和 $\mathrm{CO}$ 中毒特性. $\mathrm{Qu}$ 等 ${ }^{17}$ 采用化 学气相沉积法(CVD)制备了 $\mathrm{NG}$, 在碱性条件下不仅 具有很好的电催化氧气还原性能, 还具有良好的抗 $\mathrm{CH}_{3} \mathrm{OH}$ 和 $\mathrm{CO}$ 中毒特性. Shao 等 ${ }^{18}$ 采用 $\mathrm{N}_{2}$ 等离子体 处理热剥离的石墨烯成功制备出 $\mathrm{NG}$, 电化学测试 结果表明, 该材料对氧气还原具有较高的电催化活 性. 此后, Sheng 等 ${ }^{19}$ 采用无催化剂的热退火方法制
备出了高氮掺杂含量的石墨烯, 并在碱性介质中进 行了 ORR 测试, 结果表明该 NG 材料具有比石墨烯 更为优异的电催化性能. $\mathrm{Ma}$ 等 ${ }^{20}$ 采用爆炸辅助还原 法得到了只有几个原子层厚度的 $\mathrm{NG}$, 旋转环盘电 极(RDE)测试结果表明: 该材料的 ORR 极限扩散电 流约为没有氮掺杂石墨烯材料的两倍, 且以“直接 四电子”为主. Unni 等 ${ }^{21}$ 用吡咯作为还原剂, 首先在 $95{ }^{\circ} \mathrm{C}$ 下对 $\mathrm{GO}$ 回流 $15 \mathrm{~h}$, 然后高温处理得到吡咯- $\mathrm{N}$ (pyrrolic-N) 为主的 $\mathrm{NG}$, 该材料在 $\mathrm{O}_{2}$ 饱和 $0.1 \mathrm{~mol} \cdot \mathrm{L}^{-1}$ $\mathrm{KOH}$ 溶液中对氧的电催化还原表现出良好的循环 稳定性. Yang 等 ${ }^{22}$ 利用 $\mathrm{SiO}_{2}$ 为模版, 在 $\mathrm{NH}_{3}$ 气氛下热 处理氧化石墨烯 $(\mathrm{GO})$, 得到了 $\mathrm{N}$ 含量高达 $4.6 \%$ 的 $\mathrm{NG}, \mathrm{NG}$ 在碱性介质下对氧气进行连续阴极还原测 试 $30000 \mathrm{~s}$ 后的电流保持率为 $65 \%$, 远高于 $\mathrm{Pt} / \mathrm{C}$ $(30 \%(w))$ 的电流保持率 $(55 \%)$.

目前, NG 的合成方法主要有: CVD、 ${ }^{17}$ 电弧放 电法、 ${ }^{23}$ 溶剂热或水热反应、年在氨气气氛中热还原 $\mathrm{GO}^{25}$ 等. 三聚氰胺、 ${ }^{19}$ 尿素、 ${ }^{24}$ 丙酮肜(DMKO) ${ }^{26}$ 等常 被用作氮源对石墨烯实施氮掺杂, 其中丙酮肜作为 一种高含氮量的试剂, 具有易溶于水、毒性小、有利 于环保的特点, 是一种良好的 $\mathrm{GO}$ 还原掺杂剂. $\mathrm{Su}$ 等 ${ }^{26}$ 首次报道了利用 DMKO 作为氮源对 GO进行还 原掺杂, 将得到的 NG 用于超级电容器展示了较高 的比容量和优异的循环性能. 本文采用化学还原 法, 通过控制 DMKO 与 $\mathrm{GO}$ 量的配比制备出了具有 不同 $\mathrm{N}$ 含量的 $\mathrm{NG}$, 并对它们的物理化学性质进行 了表征, 进而研究了不同 $\mathrm{N}$ 掺杂形式及不同含量 NG 对 ORR 的电催化性能的影响.

\section{2 实验部分}

\section{1 原料与试剂}

丙酮肜(国药集团化学试剂有限公司, 化学纯), 氢氧化钾 (天津市瑞金特化学品有限公司, 分析纯), 氨水(天津市天力化学试剂有限公司, 分析纯, $28 \%$ $(w)$ ), 甲醇 (天津利安隆博华医药化学有限公司, 分 析纯), 铂炭催化剂(北京鸿海天科技有限公司, $20 \%$ $(w))$. 


\section{$2.2 \mathrm{GO}$ 分散液的制备}

氧化石墨烯通过改进 Hummers 氧化法制备, 超 声分散得到 $0.5 \mathrm{mg} \cdot \mathrm{mL}^{-1}$ 的 $\mathrm{GO}$ 分散液. ${ }^{26}$

\section{3 氮掺杂石墨烯悬浮液的制备}

在 $50 \mathrm{~mL}$ 圆底烧瓶中依次加入 $10 \mathrm{~mL} \mathrm{GO}$ 分散 液、70 $\mu \mathrm{L}$ 氨水和一定体积的丙酮肜溶液 $(14 \%(w))$; 混合均匀后, 置于电热套中, 在 $100^{\circ} \mathrm{C}$ 下加热回流 3 $h$ 后冷却至室温, 即可得到黑色的 $N G$ 分散液.

为了考察丙酩肟的用量对 $N G$ 中氮含量及其 理化性质的影响, 将在 GO 与 DMKO 的质量配比 为 $1: 0 、 1: 0.7 、 1: 1.4 、 1: 2.1$ 时得到的 $\mathrm{NG}$ 分散液分别标 记为 NG- $X(X=0,1,2,3)$.

\section{4 材料的表征及性能测试}

形貌表征采用美国 FEI 公司的 Tecnai G2 F20 STWIN 型场发射透射电子显微镜(FETEM); 紫外-可 见(UV-Vis)吸收光谱采用日本 Shimazu 公司的 UV2550 型紫外-可见分光光度计; 傅里叶变换红外(FTIR)采用 $\mathrm{KBr}$ 压片法由德国 Bruker 公司的 TENSOR27 型傅里叶变换红外光谱仪测试; zeta 电位及粒径 测试采用美国 Brookhaven 公司的 DB-525 型 zeta 电 位及粒径分析仪; 表面元素化学成分和化学态分析 采用美国 Thermo Scientific 公司的 K-Alpha 型 X射 线光电子能谱仪.

电化学测试采用三电极体系, $\mathrm{Pt}$ 丝为对电极, $\mathrm{Ag} / \mathrm{AgCl}$ 电极为参比电极, 工作电极的制备方法如 下: 将 $0.5 \mathrm{mg} \cdot \mathrm{mL}^{-1}$ 的 $\mathrm{NG}$ 或 $\mathrm{Pt} / \mathrm{C}$ 催化剂在超声清洗 器中超声 $30 \mathrm{~min}$, 然后用移液器取 $5 \mu \mathrm{L}$ 滴涂到清洁 的玻碳电极(GCE)上, 置于室温条件下 $6 \mathrm{~h}$ 干燥后待 用. 测试过程中所用的电解液为 $\mathrm{O}_{2}$ 或 $\mathrm{N}_{2}$ 饱和的 0.1 $\mathrm{mol} \cdot \mathrm{L}^{-1} \mathrm{KOH}$ 溶液, 其中循环伏安 $(\mathrm{CV})$ 测试采用上 海辰华仪器公司 CHI 660D型电化学工作站, 电位范 围为 $-1.0-+0.2 \mathrm{~V}(v \mathrm{Ag} / \mathrm{AgCl})$, 扫描速率为 $100 \mathrm{mV}$. $\mathrm{s}^{-1}$; 线性扫描伏安 (LSV)测试采用美国 BAS 公司的 BAS 100B 型旋转圆盘电极, 电位范围为 $-1.0-+0.2$ $\mathrm{V}(v s \mathrm{Ag} / \mathrm{AgCl})$, 扫描速率为 $10 \mathrm{mV} \cdot \mathrm{s}^{-1}$, 电极旋转速 率为 $900 、 1200 、 1600 、 2000 、 2500 、 3000 \mathrm{r} \cdot \mathrm{min}^{-1}$.

氧气电催化还原过程的电子转移数 $n$ 根据 Koutecky-Levich 方程中电流密度 $j^{-1}$ 与转速 $\omega^{-1 / 2}$ 的斜 率通过公式(1)、(2)计算得出.

$$
\begin{aligned}
& \frac{1}{j}=\frac{1}{j_{\mathrm{k}}}+\frac{1}{B \omega^{1 / 2}} \\
& B=0.2 n F C_{\mathrm{O}_{2}}\left(D_{\mathrm{O}_{2}}\right)^{2 / 3} v^{-1 / 6}
\end{aligned}
$$

其中 $j$ 是旋转圆盘电极的电流密度 $\left(\mathrm{A} \cdot \mathrm{dm}^{-2}\right), j_{\mathrm{k}}$ 是稳
态电流扩散密度 $\left(\mathrm{A} \cdot \mathrm{dm}^{-2}\right), \omega$ 是电极的旋转速率 $(\mathrm{r} \cdot$ $\left.\min ^{-1}\right), n$ 是一分子 $\mathrm{O}_{2}$ 还原过程中的电子转移数, $F$ 是 法拉第常数 $\left(F=96485 \mathrm{C} \cdot \mathrm{mol}^{-1}\right), D_{\mathrm{o}_{2}}$ 是 $\mathrm{O}_{2}$ 在 $0.1 \mathrm{~mol}$ $\mathrm{L}^{-1} \mathrm{KOH}$ 溶液中的扩散系数 $\left(D_{\mathrm{o}_{2}}=1.9 \times 10^{-5} \mathrm{~cm}^{2} \cdot \mathrm{s}^{-1}\right), v$ 是 $\mathrm{KOH}$ 溶液的动力学粘度 $\left(v=0.01 \mathrm{~cm}^{2} \cdot \mathrm{s}^{-1}\right), C_{\mathrm{o}_{2}}$ 是 $\mathrm{O}_{2}$ 在 $0.1 \mathrm{~mol} \cdot \mathrm{L}^{-1} \mathrm{KOH}$ 溶液中的饱和浓度 $\left(C_{\mathrm{o}_{2}}=1.2 \times 10^{-3}\right.$ $\left.\mathrm{mol} \cdot \mathrm{L}^{-1}\right)$.

\section{3 结果与讨论}

\section{1 氮掺杂石墨烯的表征}

\subsubsection{NG-1 的 TEM 分析}

图 1 所示为 NG-1 的 TEM 图像. 从图 1(a)中可以 看出, 氮掺杂石墨烯纳米片(NG-1)大部分被有效地 剥离开, 且无序地堆叠在一起, 呈透明、褶皱的薄纱 状. 这种边缘褶皱的形貌是由在对石墨烯进行剥离 和 $\mathrm{N}$ 原子掺杂过程中结构缺陷所造成的, 该缺陷结 构可以从图 1(b)的高分辨透射电镜(HRTEM)中清楚 地看出. 此外, 选区电子衍射(SAED)图中明显的六 角点阵亮斑说明所得到的 NG-1 材料具有良好的晶 格构型.

\subsubsection{UV-Vis 分析}

紫外-可见分光光谱可以用来表征 GO 的还原 过程. 如图 2(a) 所示, $\mathrm{GO}$ 在 230 和 $300 \mathrm{~nm}$ 处的吸收 峰, 分别归因于 $\mathrm{GO}$ 中芳香 $\mathrm{C}-\mathrm{C}$ 键 $\pi \rightarrow \pi^{*}$ 的电子跃 迁和 $\mathrm{C}=\mathrm{O}$ 键 $n \rightarrow \pi *$ 的电子跃迁. 当用不同量的还原 剂对 GO 进行化学还原后, 其在 $230 \mathrm{~nm}$ 处的吸收峰

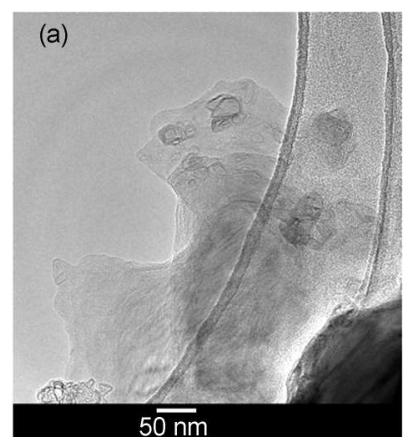

(b)

图 1 NG-1 的透射电镜(TEM) (a)及高分辨透射 电镜(HRTEM) (b)图

Fig.1 Transmission electron microscope (TEM) (a) and high resolution transmission electron microscope (HRTEM) (b) images of NG-1

NG-1: NG obtained at GO/DMKO mass ratio of 1:0.7 (NG: N-doped graphene, GO: graphene oxide, DMKO: dimethyl ketoxime). The inset in figure (b) shows the selected area electron diffraction (SAED) pattern. 

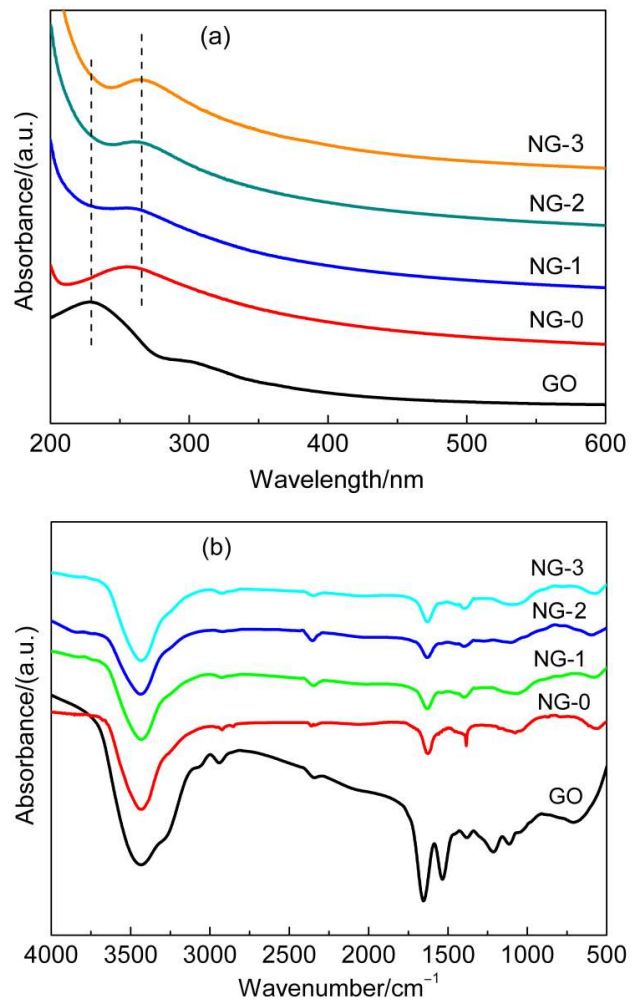

图 2 GO 与 $N G-X(X=0,1,2,3)$ 的 UV-Vis (a) 和 FTIR (b)谱图

Fig.2 UV-Vis (a) and FTIR (b) spectra of GO and NG- $X(X=0,1,2,3)$

NG- $X(X=0,1,2,3)$ denote NG obtained at GO/DMKO mass ratio of $1: 0,1: 0.7,1: 1.4,1: 2.1$, respectively.

分别发生了不同程度的红移(依次为 257、258、259 和 $261 \mathrm{~nm}$ ), 指示石墨烯的电子共轭结构得到了一 定程度的恢复, 即 GO 被 DMKO 成功地还原为石墨 烯. 由此可见, DMKO 是一种良好的还原剂, 可以有 效地对 $\mathrm{GO}$ 进行化学还原, 并且其还原程度随着还 原剂 DMKO 量的增加而逐渐增强.

\subsubsection{FTIR 分析}

图 2(b) 为 GO 与 NG- $X$ 的 FTIR 图, 可以看出: $\mathrm{GO}$ 经还原后, 对应于 3419、1670、1401、1215 和
$1123 \mathrm{~cm}^{-1}$ 附近的 $\mathrm{O}-\mathrm{H}$ 和 $\mathrm{C}=\mathrm{O}$ 键的伸缩振动、 $\mathrm{O}-$ $\mathrm{H}$ 的变形振动、 $\mathrm{C}-\mathrm{OH}$ 和 $\mathrm{C}-\mathrm{O}$ 键的伸缩振动特征 峰分别发生了减弱或消失. 另外 $\mathrm{NG}-X$ 中 $1625 \mathrm{~cm}^{-1}$ 为碳骨架振动及 $\mathrm{N}-\mathrm{H}$ 的伸缩振动, 在 $1080 \mathrm{~cm}^{-1}$ 新 出现了 $\mathrm{C}-\mathrm{N}$ 键的弱伸缩运动.

\subsubsection{Zeta电位及粒径分析}

Zeta 电位和粒径分析可以用来表征 $\mathrm{GO}$ 与 $\mathrm{NG}-$ $X$ 的表面所带电荷及粒径的大小. 如图 3 所示, $\mathrm{GO}$ 的 zeta 电位为 $-45 \mathrm{mV}$, 说明 $\mathrm{GO}$ 表面含有大量的负 电荷基团, 如 $-\mathrm{COO}^{-} 、-\mathrm{O}^{-}$等含氧官能基团. 随着 DMKO 量的增加, DMKO 对 GO 还原程度越高, 其 表面的含氧基团减少的越多, 导致石墨烯表面 zeta 电位正移, 其片层之间的静电排斥力减弱而发生聚 集, 粒径逐渐增大. 这点可以从XPS 分析结果(表 1) 中 $\mathrm{O}$ 含量的变化得到证实. 当 $\mathrm{GO}$ 与 $\mathrm{DMKO}$ 的质量 比为 $1: 2.1$ 时, 其粒径达到了 $800 \mathrm{~nm}$.

\section{1 .5 XPS 分析}

为了考察 $\mathrm{DMKO}$ 对 $\mathrm{GO}$ 的还原与掺杂作用, 利 用 XPS 对 GO 与 NG- $X$ 进行了表面组成分析. 图 4(a) 为 $\mathrm{GO}$ 和 $\mathrm{NG}-X$ 的 XPS 全谱图, 可以看出 $\mathrm{GO}$ 中只含

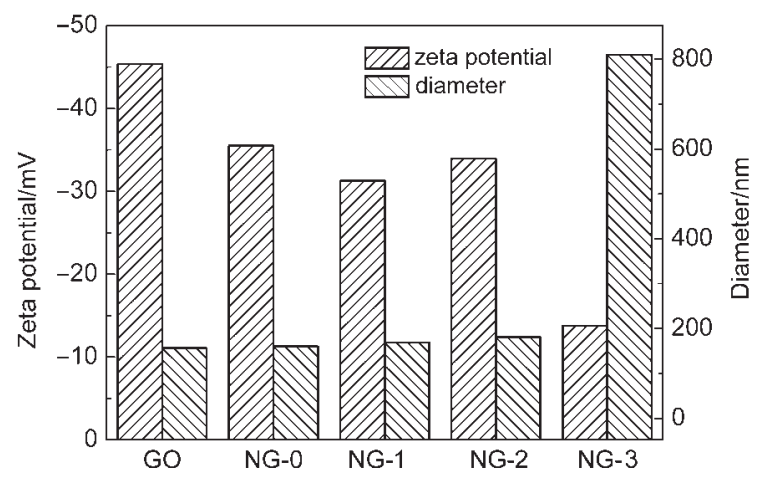

图 $3 \mathrm{GO}$ 与 $\mathrm{NG}-X(X=0,1,2,3)$ 的 zeta 电位及 粒径分析图

Fig.3 Zeta potential and nano-particle analysis of GO and NG- $X(X=0,1,2,3)$

表 1 GO 和 NG- $X$ 的XPS 定量分析及其催化氧还原反应的电子转移数

Table 1 Quantitative analysis of XPS data of GO and NG- $X$, and the transferred electron numbers for catalysis $\mathrm{O}_{2}$ reduction reaction

\begin{tabular}{|c|c|c|c|c|c|c|c|c|c|}
\hline \multirow{2}{*}{ Sample } & \multicolumn{2}{|r|}{$\mathrm{C} 1 s$} & \multicolumn{2}{|r|}{$\mathrm{O} 1 s$} & \multicolumn{2}{|r|}{$\mathrm{N} 1 s$} & \multicolumn{2}{|c|}{ Atomic ratio } & \multirow{2}{*}{$n$} \\
\hline & $E_{\mathrm{B}} / \mathrm{eV}$ & Atomic fraction/\% & $E_{\mathrm{B}} / \mathrm{eV}$ & Atomic fraction/\% & $E_{\mathrm{B}} / \mathrm{eV}$ & Atomic fraction/\% & $\mathrm{C} / \mathrm{O}$ & $\mathrm{C} / \mathrm{N}$ & \\
\hline GO & 285.9 & 67.79 & 532.3 & 32.21 & - & - & 2.10 & - & - \\
\hline NG-0 & 285.2 & 82.42 & 532.3 & 13.18 & 399.7 & 4.40 & 6.25 & 18.73 & 2.2 \\
\hline NG-1 & 285.3 & 82.15 & 532.5 & 12.78 & 400.0 & 5.07 & 6.42 & 16.20 & 3.6 \\
\hline NG-2 & 286.5 & 81.94 & 533.2 & 12.17 & 400.0 & 5.89 & 6.73 & 13.91 & 3.2 \\
\hline NG-3 & 285.1 & 83.16 & 532.5 & 11.83 & 399.8 & 5.01 & 7.03 & 16.60 & 2.4 \\
\hline
\end{tabular}



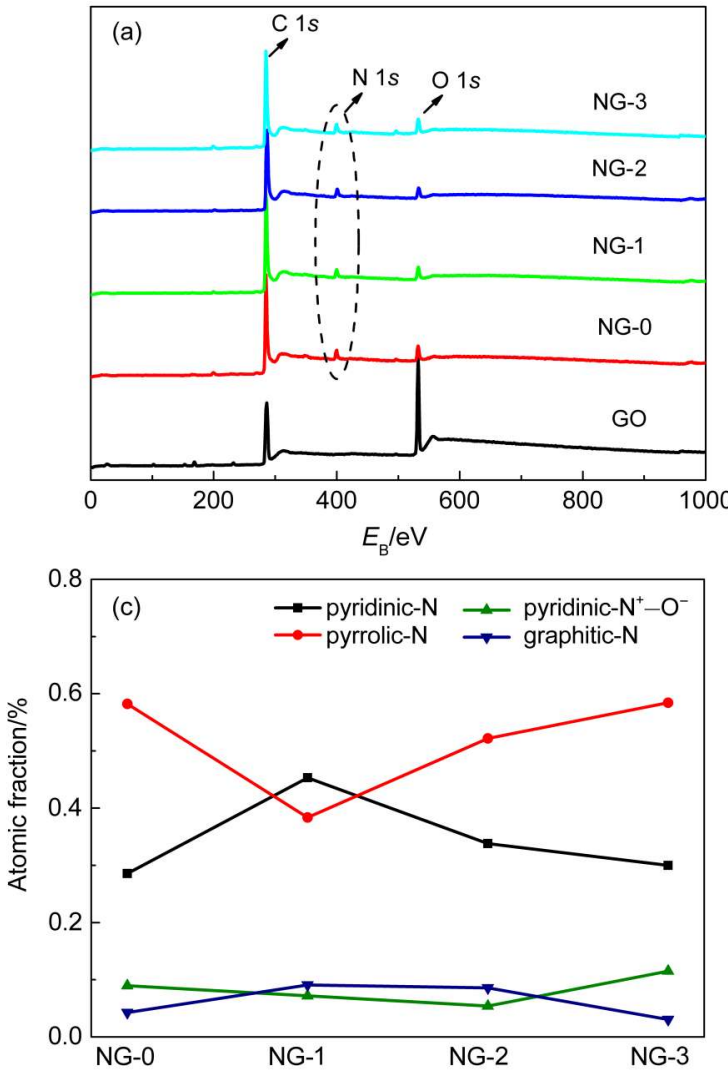
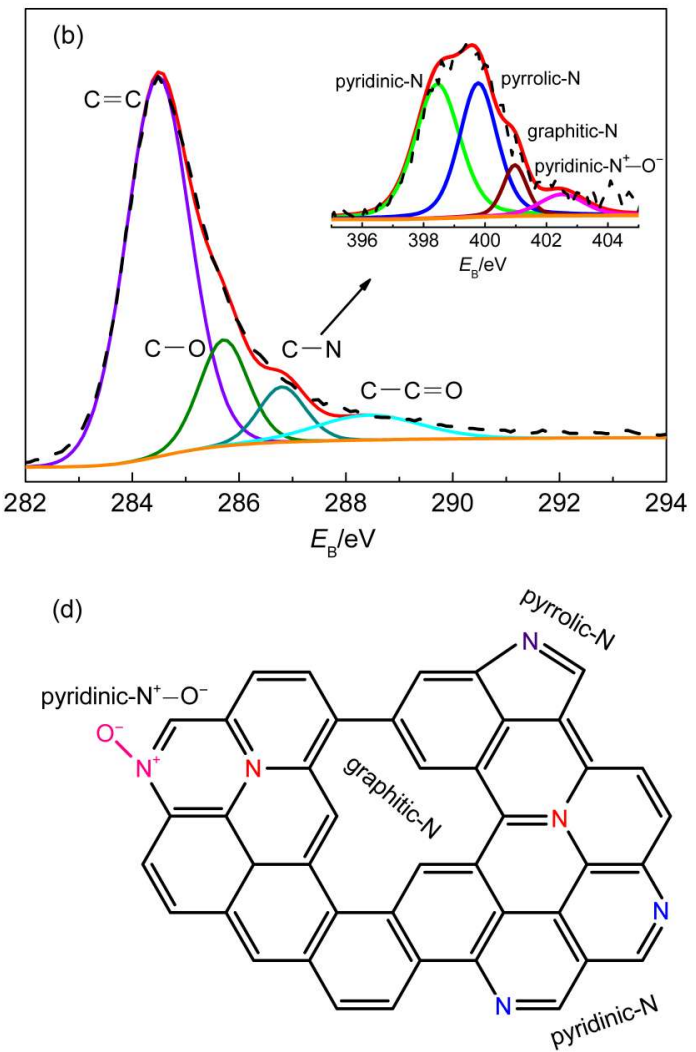

图 4 (a) GO 与 NG- $X$ 的全谱图; (b) NG-1 的高分辨C 1s XPS 谱; (c) NG- $X$ 不同 $\mathrm{N}$ 掺杂形式的原子含量;

(d) NG 的结构示意图

Fig.4 (a) XPS survey scan spectra of GO and NG-X; (b) high resolution XPS C $1 \mathrm{~s}$ spectra of NG-1; (c) atom content of different doping nitrogen of NG- $X$; (d) schematic representation of NG The inset in figure (b) shows the high resolution XPS N $1 s$ spectra of NG-1.

有 $\mathrm{C}$ 和 $\mathrm{O}$ 原子, 且 $\mathrm{C} / \mathrm{O}$ 的原子比为 2.10 ; 而 $\mathrm{GO}$ 经 $\mathrm{DMKO}$ 还原后, 其 $\mathrm{C} / \mathrm{O}$ 原子比增加到 6.25-7.03. 此 外, $\mathrm{NG}$ 在 $399.5 \mathrm{eV}$ 处出现了一个 $\mathrm{N} 1 s$ 峰, 说明 $\mathrm{DMKO}$ 在对 $\mathrm{GO}$ 进行还原的同时还实现了氮掺杂. 而在对 $\mathrm{NG}-X$ 进行组成分析时发现: 随着 $\mathrm{DMKO}$ 量 的增加, $\mathrm{NG}$ 中 $\mathrm{O}$ 原子含量逐渐减少, 而 $\mathrm{N}$ 原子含量 先增加后减小, 在 NG-2 中氮含量达到最高(表 1).

此外, 在对 $\mathrm{NG}-X$ 的 $\mathrm{C} 1 s$ 和 $\mathrm{N} 1 s$ 进行了分峰拟 合时发现: NG-1 结构中的 C 主要以 $\mathrm{C}-\mathrm{C}$ 或 $\mathrm{C}=\mathrm{C}$ $(\sim 284.8 \mathrm{eV}) 、 \mathrm{C}-\mathrm{OH}(\sim 285.9 \mathrm{eV}) 、 \mathrm{C}-\mathrm{N}(\sim 287.5 \mathrm{eV})$ 和 $\mathrm{O}-\mathrm{C}=\mathrm{O}(\sim 289.0 \mathrm{eV})$ 的形式存在, ${ }^{27}$ 见图 4(b). 而 $\mathrm{N} 1 s$ 则以以下四种形式存在 (图 4(d)), 即吡啶 $-\mathrm{N}$ (pyridinic-N, $398.2 \mathrm{eV}$ )、吡咯-N (pyrrolic-N, 400.0 $\mathrm{eV}$ )、石墨化 $-\mathrm{N}$ (graphitic- $\mathrm{N}, \sim 401.0 \mathrm{eV}$ ) 和吡啶 $-\mathrm{O}$ (pyridinic- $\mathrm{N}^{+}-\mathrm{O}^{-}, \sim 403.0 \mathrm{eV}$ ). ${ }^{28}$ 图 4(c) 为 $\mathrm{NG}-X$ 的不 同氮掺杂形式含量, 可以看出, DMKO 的量对不同 掺杂形式氮的含量具有很大的影响, 其中 NG-1 中 的吡啶-N含量最高, 这对于电催化氧还原性能具有
重要影响.

\section{2 氮掺杂石墨烯的电催化氧还原性能测试}

\subsection{1 氮掺杂石墨烯的 ORR 性能}

图 5(a) 和 5(b) 所示分别为 GCE 和 NG-1 的 CV 曲 线. 对比裸 GCE 电极, NG-1 的 ORR 起峰电位的正 移和峰电流的增加, 说明氧还原反应在该材料表面 具有更快的电子转移速率和更高的反应活性. 此 外, 与 $\mathrm{GO}$ 相比 $\mathrm{NG}-X$ 对 ORR 表现出更强的催化活 性(图 5(c)), 这是由于 $\mathrm{GO}$ 经还原之后, $\mathrm{NG}-X$ 导电性 增强, 增加了 $\mathrm{ORR}$ 电子转移速率; 同时 $\mathrm{N}$ 原子的引 入增加了其缺陷结构和活性位点. 对比 NG- $X$ 的起 峰电位和峰电流与图 4(c)中吡啶-N含量之间的关系 发现: 吡啶- $\mathrm{N}$ 含量直接影响着 $\mathrm{NG}$ 的氧催化还原活 性大小. 这主要归因于吡啶- $\mathrm{N}$ 具有较强的吸电子能 力, 导致与之相邻的 $\mathrm{C}$ 原子表面带部分正电荷, 从而 有利于 $\mathrm{O}_{2}$ 的吸附和还原. 相对于 NG-0, 虽然 NG-3 的吡啶-N含量较高, 但其在溶液中发生团聚减少了 石墨烯的边缘缺陷和活性位点, 从而导致其 ORR 催 

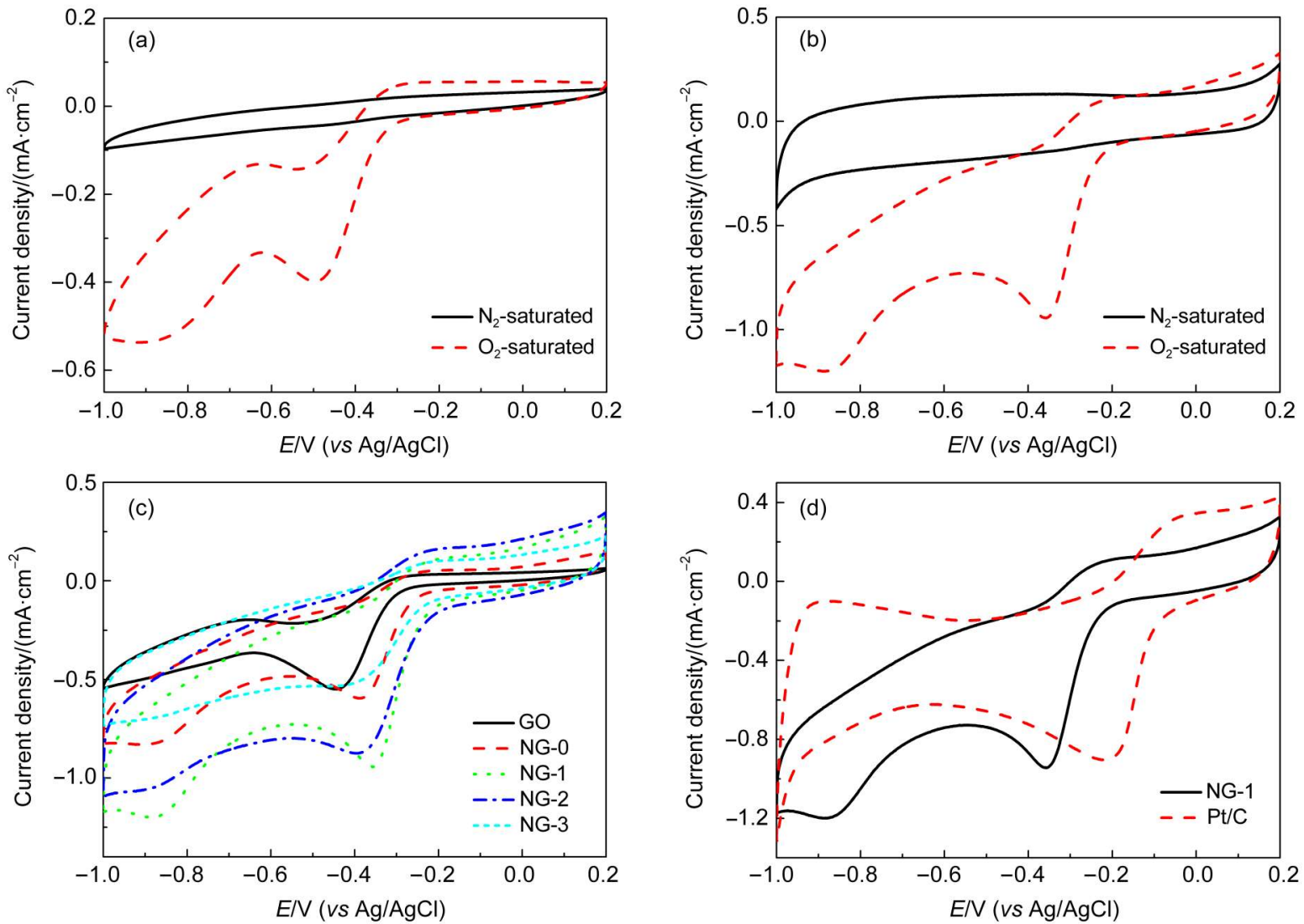

图 5 GCE (a), NG-1 (b), GO 与 NG- $X(c), N G-1$ 和 Pt/C (d) 在 $\mathrm{O}_{2}$ (或 $\mathrm{N}_{2}$ )饱和的 $0.1 \mathrm{~mol} \cdot \mathrm{L}^{-1} \mathrm{KOH}$ 溶液中的 $\mathrm{CV}$ 曲线

Fig.5 CV curves of bare GCE (a), NG-1 (b), GO and NG- $X$ (c), NG-1 and Pt/C (d) in an $\mathrm{O}_{2}$ (or $\mathrm{N}_{2}$ )-saturated

$0.1 \mathrm{~mol} \cdot \mathrm{L}^{-1} \mathrm{KOH}$ solution

scan rate: $100 \mathrm{mV} \cdot \mathrm{s}^{-1}$

化活性的降低.

起峰电位和电流密度是评价电催化剂性能的 指标之一. 图 5(d)为 NG-1 与商用 $\mathrm{Pt} / \mathrm{C}$ 对 $\mathrm{ORR}$ 的 $\mathrm{CV}$ 曲线, $\mathrm{Pt} / \mathrm{C}$ 催化剂的起峰电位约为 $-0.1 \mathrm{~V}$, 电流密度 约为 $0.9 \mathrm{~mA} \cdot \mathrm{cm}^{-2}$. 相对于 $\mathrm{Pt} / \mathrm{C}$ 催化剂, NG-1 虽然起 峰电位较负(约 $-0.2 \mathrm{~V}$ ), 但其电流密度(约 $-0.96 \mathrm{mV}$. $\mathrm{cm}^{-2}$ ) 有一定的提高. 此外, 我们还研究了 NG-1 修饰

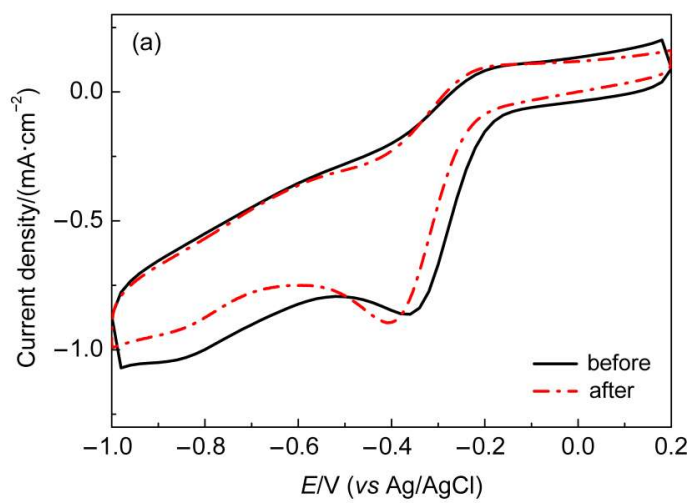

电极催化氧还原反应稳定性(图 6). 可以看出, 经过 10000 次 ORR 循环, 虽然 $\mathrm{Pt} / \mathrm{C}$ 起峰电位没有改变, 但其电流密度却降低了近 50\%. 而 NG-1 在循环前 后电流密度基本没有变化, 仅起峰电位向负移动了 $0.04 \mathrm{~V}$. 以上结果表明, NG-1 具有比 $\mathrm{Pt} / \mathrm{C}$ 催化剂更 优异的电催化稳定性和循环寿命, 其在燃料电池领 域将具有非常广阔的应用前景.

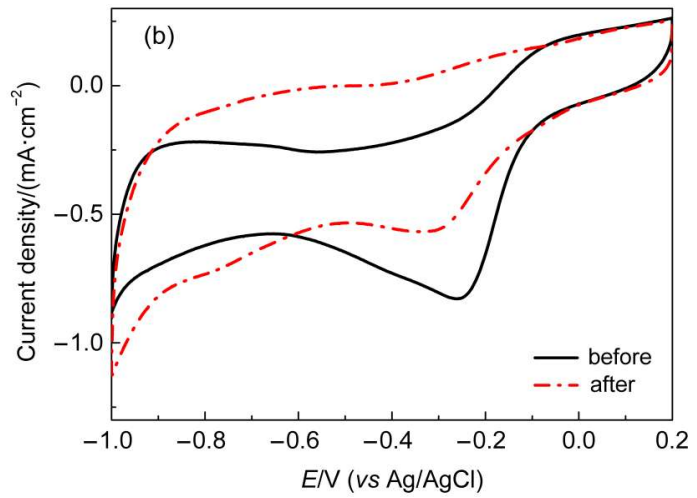

图 6 NG-1 (a) 和 Pt/C (b) 修饰电极在 $\mathrm{O}_{2}$ 饱和 $0.1 \mathrm{~mol} \cdot \mathrm{L}^{-1} \mathrm{KOH}$ 溶液中循环扫描 10000 次前后的 $\mathrm{CV}$ 曲线

Fig.6 CV curves of NG-1 (a) and $\mathrm{Pt} / \mathrm{C}$ (b) before and after 10000 cycles in $\mathrm{O}_{2}$-saturated $0.1 \mathrm{~mol} \cdot \mathrm{L}^{-1} \mathrm{KOH}$ solutions 


\subsection{2 氮掺杂石墨烯的 RDE 测试}

虽然 CV 技术在一定程度上能够反映 $\mathrm{NG}$ 的氧 催化还原性能, 但却不能对其进行完整的评价, 因 此选择 RDE 对该材料的 ORR 反应机理进行进一步 的探讨. 图 7(a)所示为 NG-1 在不同转速下的线性扫 描伏安曲线(LSV), 可以看出电流密度随着转速的 增加而增大, 其 $-0.2 \mathrm{~V}$ 的起峰电位与图 5(b)中测试 结果相符. 利用电流密度 $j^{-1}$ 与转速 $\omega^{-0.5}$ 之间的线性 关系, 结合 Koutecky-Levich 方程进行计算其 ORR 中 $\mathrm{O}_{2}$ 还原的电子转移数. 图 7(a)插图显示在 $-0.4-$ $-0.7 \mathrm{~V}$ 电压范围内 $j^{-1}$ 与 $\omega^{-0.5}$ 之间呈良好的线性关 系, 说明 ORR 反应在该材料表面的电子转移数基本 相同. 根据公式(1)和(2)计算得出其在 $-0.4--0.7 \mathrm{~V}$ 电压范围内的电子转移数 $n$ 为 3.4-3.8, 表明 $\mathrm{O}_{2}$ 在该 材料表面的电催化是一个 “直接四电子” 过程 $\left(\mathrm{O}_{2}+\right.$ $\left.2 \mathrm{H}_{2} \mathrm{O}+4 \mathrm{e}^{-} \rightarrow 4 \mathrm{OH}^{-}\right)$.

在 $1600 \mathrm{r} \cdot \mathrm{min}^{-1}$ 转速条件下分别对 $\mathrm{NG}-X$ 进行
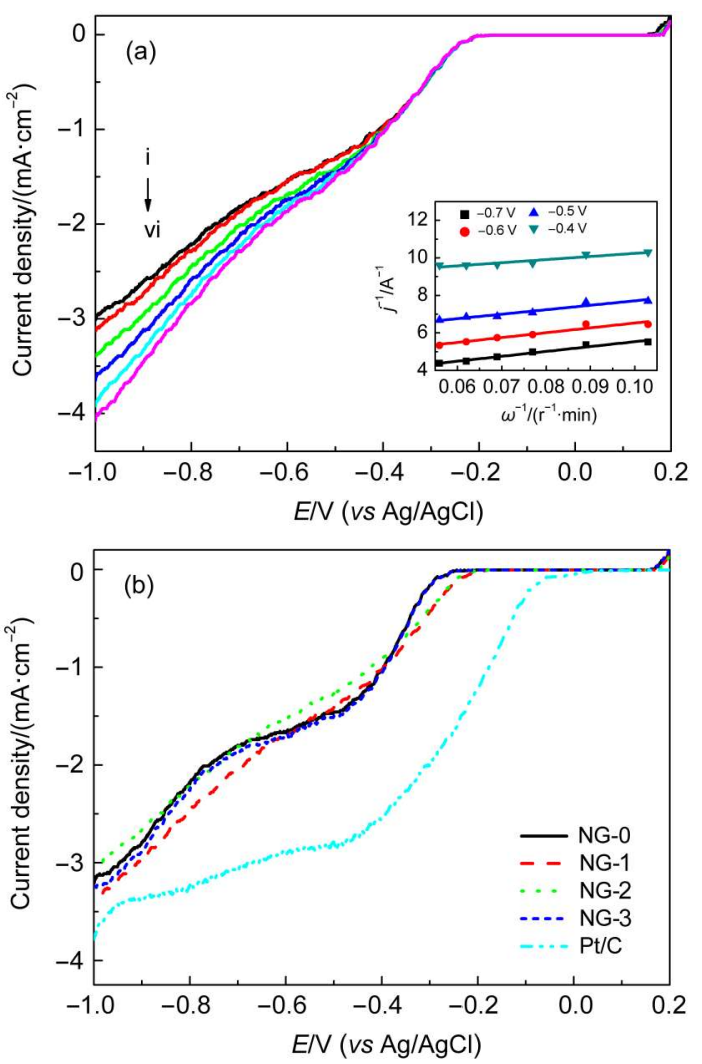

图 7 (a) NG-1 在不同转速下 LSV 曲线; (b) 在 $\mathrm{O}_{2}$ 饱和 0.1 $\mathrm{mol} \cdot \mathrm{L}^{-1} \mathrm{KOH}$ 中 $\mathrm{NG}-X$ 与 $\mathrm{Pt} / \mathrm{C}$ 在 $1600 \mathrm{r} \cdot \mathrm{min}^{-1}$ 的 $\mathrm{LSV}$ 曲线

Fig.7 (a) LSV curves recorded for NG-1 with different rotation rates; (b) LSV curves for NG- $X$ and $\mathrm{Pt} / \mathrm{C}$ in an $\mathrm{O}_{2}$-saturated $0.1 \mathrm{~mol} \cdot \mathrm{L}^{-1} \mathrm{KOH}$ solution $\left(1600 \mathrm{r} \cdot \mathrm{min}^{-1}\right)$ rotation rate in (a) from $i$ to vi: 900, 1200, 1600, 2000, 2500, $3000 \mathrm{r} \cdot \mathrm{min}^{-1}$; scan rate: $100 \mathrm{mV} \cdot \mathrm{s}^{-1} ; \mathrm{LSV}$ : linear scanning voltammetry
旋转圆盘电极的测试(图 7(b)), 进而考察不同氮掺 杂形式对 ORR 的电子转移数的影响 (表 1). 从图 中明显看出: NG-0 与 NG-3 的氧催化是一个两步 还原的过程, 且其起峰电位分别为 $-0.25 、-0.70$ $\mathrm{V}$ 与 $-0.25 、-0.72 \mathrm{~V}$. 说明 $\mathrm{ORR}$ 在该材料的表面是一 个两步两电子的过程 (NG-0 与 NG-3 的 $n$ 值为 2.2 与 2.4 ), 而 NG-2 的电子转移数 $n=3.2$. 结合图 4(c) 中吡 啶- $\mathrm{N}$ 含量说明吡啶 $-\mathrm{N}$ 影响着 ORR 过程的电子转移 数 $n$.

据实验及理论计算表明: $:^{29,30}$ 石墨 $-\mathrm{N}$ 和吡啶 $-\mathrm{N}$ 是氧发生电化学还原的活性位点. 理论计算还认 为, 石墨化- $\mathrm{N}$ 的氧还原超电势较吡啶 $\mathrm{N}$ 的大; 根据 过氧化反应机理, 吡啶-N位点不利于发生 $\mathrm{O}_{2} \rightarrow \mathrm{HO}_{2}^{-}$ 的吸附反应, ${ }^{30}$ 而倾向发生直接“四电子过程”. 此 外, 石墨化- $\mathrm{N}$ 相对于吡啶- $\mathrm{N}$ 和吡咯- $\mathrm{N}$ 具有更高的 氧还原催化活性和大的电流密度. ${ }^{31}$

NG- $X(X=0,1,2,3)$ 等材料对催化氧还原时的 电子转移数分别为 $2.2 、 3.6 、 3.2 、 2.4$. 通过分析 $\mathrm{NG}-X$ 等材料中不同 $\mathrm{N}$ 掺杂形式的原子含量(图 4(c)), 可以
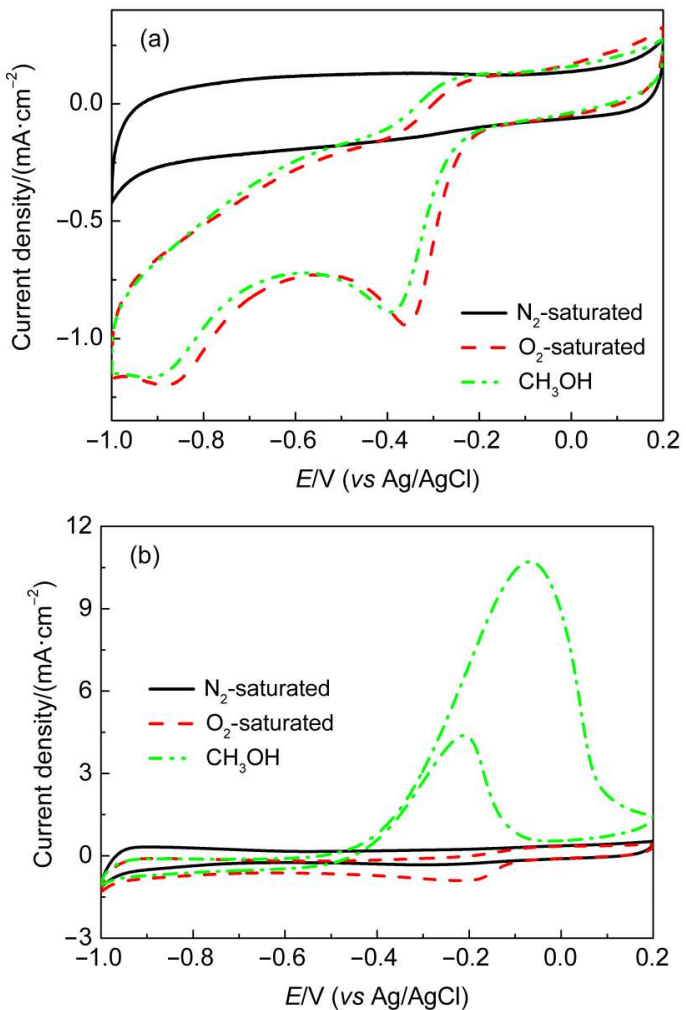

图 8 在 $\mathrm{O}_{2}$ 饱和 $0.1 \mathrm{~mol} \cdot \mathrm{L}^{-1} \mathrm{KOH}$ 中加入 $3 \mathrm{~mol} \cdot \mathrm{L}^{-1}$ 甲醇 前后 NG-1 (a) 和 Pt/C (b) 的 CV 曲线

Fig.8 CV curves of NG-1 (a) and $\mathrm{Pt} / \mathrm{C}$ (b) in $\mathrm{O}_{2}$-saturated $0.1 \mathrm{~mol} \cdot \mathrm{L}^{-1} \mathrm{KOH}$ solutions with and without $3 \mathrm{~mol} \cdot \mathrm{L}^{-1} \mathrm{CH}_{3} \mathrm{OH}$ scan rate: $100 \mathrm{mV} \cdot \mathrm{s}^{-1}$ 
看出: 石墨化- $\mathrm{N}$ 、吡啶- $\mathrm{N}$ 和吡咯- $\mathrm{N}$ 对电子转移数都 有较大的影响, 其中石墨化- $\mathrm{N}$ 和吡啶-N对氧发生 “四电子”还原过程有利, 而吡咯-N对氧发生“二电 子”还原过程有利. 由于材料 $\mathrm{NG}-X$ 中石墨化- $\mathrm{N}$ 、吡 啶- $\mathrm{N}$ 和吡咯- $\mathrm{N}$ 含量的差异, 造成 $\mathrm{NG}-X$ 的电子转移 数差异较大.

另外, 通过对比 $\mathrm{NG}-X$ 等材料中不同 $\mathrm{N}$ 掺杂形 式的原子含量(图 4(c)) 及其在 $\mathrm{O}_{2}$ 饱和的 $0.1 \mathrm{~mol} \cdot \mathrm{L}^{-1}$ $\mathrm{KOH}$ 溶液中 $\mathrm{CV}$ 曲线(图 5(c)), 可以看出: 石墨化-N、 吡啶-N 和吡咯-N 对峰电位和峰电流有较大的影响, 其中石墨化-N由于其较高的电子导电性倾向于产 生较高的氧还原峰电流, 而吡啶- $\mathrm{N}$ 较低的超电势倾 向于产生较正的氧还原峰电位.

\subsubsection{NG-1与 $\mathrm{Pt} / \mathrm{C}$ 的抗甲醇效应}

在 $\mathrm{O}_{2}$ 饱和 $0.1 \mathrm{~mol} \cdot \mathrm{L}^{-1} \mathrm{KOH}$ 溶液中通过对比加 入 $3 \mathrm{~mol} \cdot \mathrm{L}^{-1}$ 甲醇前后 $\mathrm{NG}-1$ 与 $\mathrm{Pt} / \mathrm{C}$ 的 $\mathrm{CV}$ 曲线来考 察其抗甲醇“跨界效应”(图 8). 可以看出, 在加入 3 $\mathrm{mol} \cdot \mathrm{L}^{-1}$ 甲醇后 $\mathrm{NG}-1$ 氧催化还原活性几乎没有变 化; 而 $\mathrm{Pt} / \mathrm{C}$ 催化剂在 -0.1 和 $-0.2 \mathrm{~V}$ 附近出现了一对 $\mathrm{CH}_{3} \mathrm{OH}$ 氧化还原峰, 同时 $-0.2 \mathrm{~V}$ 左右的氧还原峰消 失了. 由此得出, NG-1 具有比 Pt/C 催化剂更优异的 ORR 选择性和抗 $\mathrm{CH}_{3} \mathrm{OH}$ “跨界效应”特性. 综上所 述, 氮掺杂石墨烯材料对氧还原反应表现出优异的 电催化性能, 并具有良好的抗甲醇特性, 有望应用 于直接甲醇燃料电池和碱性燃料电池中.

\section{4 结 论}

分别以 $G O$ 和 DMKO 为碳源和氮源, 通过调节 两者的质量比, 利用化学还原法在 $100{ }^{\circ} \mathrm{C}$ 下回流 $3 \mathrm{~h}$ 得到了氮含量为 $4.40 \%-5.89 \%$ 的 NG. 其中氮原子 分别以吡啶 $-\mathrm{N}$ 、吡啶- $\mathrm{N}$ 、吡啶 $-\mathrm{N}$ 和吡啶 $-\mathrm{N}^{+}-\mathrm{O}^{-}$的形 式掺杂进入石墨烯结构中, 且不同材料的相对含量 也有明显的差别. 电化学测试表明: 在 $\mathrm{O}_{2}$ 饱和的 0.1 $\mathrm{mol} \cdot \mathrm{L}^{-1} \mathrm{KOH}$ 溶液中吡啶 $-\mathrm{N}$ 含量最高的 NG- 1 表现 出优异的 ORR 活性, 其氧还原电子转移数为 3.6. 此 外, 该材料还具有良好的抗 $\mathrm{CH}_{3} \mathrm{OH}^{\circ}$ “跨界效应” 的特 性, 这归因于吡啶- $\mathrm{N}$ 的存在可以使相邻的 $\mathrm{C}$ 原子表 面带部分正电荷, 从而有助于 $\mathrm{O}_{2}$ 的吸附和还原. 此 外, 石墨化-N由于其较高的电子导电性倾向于产生 较高的氧还原峰电流, 而吡啶- $\mathrm{N}$ 较低的超电势倾向 于产生较正的氧还原峰电位.

致谢：感谢陕西师范大学漆红兰副教授对本文旋转圆盘电
极实验提供帮助.

\section{References}

(1) Debe, M. K. Nature 2012, 486, 43. doi: 10.1038/nature11115

(2) Yang, R.; Leisch, J.; Strasser, P.; Toney, M. F. Chem. Mater. 2010, 22, 4712. doi: 10.1021/cm101090p

(3) Chen, A.; Holt-Hindle, P. Chem. Rev. 2010, 110, 3767. doi: $10.1021 / \mathrm{cr} 9003902$

(4) Zheng, Y.; Jiao, Y.; Jaroniec, M.; Jin, Y.; Qiao, S. Z. Small 2012, 8,3550 .

(5) Zhang, L.; Zhang, J.; Wilkinson, D. P.; Wang, H. J. Power Sources 2006, 156, 171. doi: 10.1016/j.jpowsour.2005.05.069

(6) Zhang, M.; Dai, L. Nano Energy, 2012, 1, 514. doi: 10.1016/j. nanoen.2012.02.008

(7) Nallathambi, V.; Lee, J. W.; Kumaraguru, S. P.; Wu, G.; Popov, B. N. J. Power Sources 2008, 183, 34. doi: 10.1016/j. jpowsour.2008.05.020

(8) Hu, Y. J.; Jin, J.; Zhang, H.; Wu, P.; Cai, C. X. Acta Phys. -Chim. Sin. 2010, 26, 2073. [胡耀娟, 金 娟, 张 卉, 吴 萍, 蔡称 心. 物理化学学报, 2010, 26, 2073.] doi: 10.3866/PKU. WHXB20100812

(9) Bunch, J. S.; Verbridge, S. S.; Alden, J. S.; van der Zande, A. M.; Parpia, J. M.; Craighead, H. G.; McEuen, P. L. Nano Lett. 2008, 8, 2458. doi: 10.1021/n1801457b

(10) Park, S.; Ruoff, R. S. Nat. Nanotechnol. 2009, 4, 217. doi: 10.1038/nnano.2009.58

(11) Novoselov, K. S.; Jiang, Z.; Zhang, Y.; Morozov, S. V.; Stormer, H. L.; Zeitler, U.; Maan, J. C.; Boebinger, G. S.; Kim, P.; Geim, A. K. Science 2007, 315, 1379. doi: 10.1126/science.1137201

(12) Balandin, A. A.; Ghosh, S.; Bao, W.; Calizo, I.; Teweldebrhan, D.; Miao, F.; Lau, C. N. Nano Lett. 2008, 8, 902. doi:10.1021/ n10731872 doi: 10.1021/n10731872

(13) Avouris, P.; Chen, Z.; Perebeinos, V. Nat. Nanotechnol. 2007, 2, 605. doi: 10.1038/nnano.2007.300

(14) Wang, X.; Li, X.; Zhang, L.; Yoon, Y.; Weber, P. K.; Wang, H.; Guo, J.; Dai, H. Science 2009, 324, 768. doi: 10.1126/ science. 1170335

(15) Jeon, I. Y.; Choi, H. J.; Choi, M.; Seo, J. M.; Jung, S. M.; Kim, M. J.; Zhang, S.; Zhang, L.; Xia, Z.; Dai, L.; Park, N.; Baek, J. B. Scientific Reports 2013, 3, 1810.

(16) Geng, D.; Chen, Y.; Chen, Y.; Li, Y.; Li, R.; Sun, X.; Ye, S.; Knights, S. Energy Environ. Sci. 2011, 4, 760. doi: 10.1039/ c0ee00326c

(17) Qu, L.; Liu, Y.; Baek, J. B.; Dai, L. ACS Nano 2010, 4, 1321. doi: 10.1021/nn901850u

(18) Shao, Y.; Zhang, S.; Engelhard, M. H.; Li, G.; Shao, G.; Wang, Y.; Liu, J.; Aksay, I. A.; Lin, Y. J. Mater. Chem. 2010, 20, 7491. doi: $10.1039 / \mathrm{c} 0 \mathrm{jm} 00782 \mathrm{j}$

(19) Sheng, Z. H.; Shao, L.; Chen, J. J.; Bao, W. J.; Wang, F. B.; Xia, X. H. ACS Nano 2011, 5, 4350. doi: 10.1021/nn103584t

(20) Ma, G. X.; Zhao, J. H.; Zheng, J. F.; Zhu, Z. P. New Carbon 
Mater. 2012, 27, 258. [马贵香, 赵江红, 郑剑锋, 朱珍平. 新型 炭材料, 2012, 27, 258.]

(21) Unni, S. M.; Devulapally, S.; Karjule, N.; Kurungot, S. J. Mater. Chem. 2012, 22, 23506. doi: 10.1039/c2jm35547g

(22) Yang, S.; Zhi, L.; Tang, K.; Feng, X.; Maier, J.; Müllen, K. Adv. Funct. Mater. 2012, 22, 3634. doi: 10.1002/adfm.v22.17

(23) Li, N.; Wang, Z.; Zhao, K.; Shi, Z.; Gu, Z.; Xu, S. Carbon 2010, 48, 255. doi: 10.1016/j.carbon.2009.09.013

(24) Su, P.; Guo, H. L.; Peng, S.; Ning, S. K. Acta Phys. -Chim. Sin. 2012, 28, 2745. [苏 鹏, 郭慧林, 彭 三, 宁生科. 物理化学 学报, 2012, 28, 2745.] doi: 10.3866/PKU.WHXB201208221

(25) Li, X.; Wang, H.; Robinson, J. T.; Sanchez, H.; Diankov, G.; Dai, H. J. Am. Chem. Soc. 2009, 131, 15939. doi: 10.1021/ ja907098f
(26) Su, P.; Guo, H. L.; Tian, L.; Ning, S. K. Carbon 2012, 50, 5351. doi: 10.1016/j.carbon.2012.07.001

(27) Wang, Y.; Shao, Y.; Matson, D. W.; Li, J.; Lin, Y. ACS Nano 2010, 4, 1790. doi: 10.1021/nn100315s

(28) Lin, Z.; Waller, G. H.; Liu, Y.; Liu, M.; Wong, C. P. Carbon 2013, 53, 130. doi: 10.1016/j.carbon.2012.10.039

(29) Subramanian, N. P.; Li, X.; Nallathambi, V.; Kumaraguru, S. P.; Colon-Mercado, H.; Wu, G.; Lee, J W.; Popov, B. N. J. Power Sources 2009, 188, 38. doi: 10.1016/j.jpowsour.2008.11.087

(30) Saidi, W. A. J. Phys. Chem. Lett. 2013, 4, 4160. doi: 10.1021/ jz402090d

(31) Lai, L.; Potts, J. R.; Zhan, D.; Wang, L.; Poh, C. K.; Tang, C.; Gong, H.; Shen, Z.; Lin, J.; Ruoff, R. S. Energy Environ. Sci. 2012, 5, 7936. doi: 10.1039/c2ee21802j 